\title{
We must ask relevant questions and answer with meaningful outcomes
}

\author{
Ronald B. George, MD, FRCPC • Dolores M. McKeen, MD, MSc, FRCPC
}

Received: 30 May 2017/ Accepted: 12 June 2017/Published online: 16 June 2017

(C) Canadian Anesthesiologists' Society 2017

"Nobody knew that health care could be so complicated", is how United States President Donald Trump portrayed his first month's experience with healthcare policy when he addressed a group of governors at the White House on February 27, 2017. ${ }^{1}$

Most anybody working in healthcare could have let President Trump in on our poorly kept secret - healthcare is complicated! In fact, healthcare is very complicated, and in specific situations, a lack of standardized end points and patient-centred outcome measures can contribute to this complicated state of affairs. Relevant patient-centred outcome measures should be validated, have clinical meaning (i.e., personal value to patients), and result in improved care or facilitate positive healthcare experience but in certain circumstances, they can get complicated.

In this issue of the Journal, Desgranges et al. ${ }^{2}$ present the findings of their prospective cohort study examining the predictive factors of maternal hypothermia during Cesarean delivery. In their study, the authors monitored the tympanic temperatures of women who were scheduled for either elective or emergency Cesarean delivery. Temperature was measured upon arrival in the operating room, at skin incision, and at the end of the skin closure. Cesarean deliveries performed under either neuraxial or general anesthesia were included.

After induction of anesthesia, patients were kept warm with a cotton blanket over their upper body and with an upper body forced-air warmer set at $43^{\circ} \mathrm{C}$. Hypothermia

\footnotetext{
R. B. George, MD, FRCPC - D. M. McKeen, MD, MSc, FRCPC $(\square)$

Department of Women's \& Obstetric Anesthesia, IWK Health

Centre, Dalhousie University, 5850/5980 University Avenue,

P.O. Box 9700, Halifax, NS B3K 6R8, Canada

e-mail: D.McKeen@Dal.Ca
}

was defined as a tympanic temperature $<36^{\circ} \mathrm{C}$ at the end of the skin suture. Various demographic, preoperative, and intraoperative surgical and anesthetic factors were recorded and analyzed as possible predictors of maternal hypothermia. Three hundred fifty-nine women were included in the analysis, and $23 \%$ of these patients experienced hypothermia. The investigators' analysis showed that the use of active forced-air warming was associated with a decreased risk for maternal hypothermia, while a lower maternal temperature upon arrival in the operating room as well as an increased volume of unwarmed intravenous fluids were predictors of maternal hypothermia. Desgranges et al. suggest their results should encourage each anesthesiologist to monitor maternal temperature before and during Cesarean delivery, since maternal hypothermia may lead to several complications.

Nevertheless, readers should consider the following fundamental question after reading the Desgranges et al. ${ }^{2}$ study: What is the relevance of the clinical problem of maternal hypothermia during Cesarean delivery with regard to the patient? Applying the FINER criteria (feasible, interesting, novel, ethical, and relevant) ${ }^{3}$ clinical researchers seek to provide answers. When presented with clinical problems, we ask questions, explain the facts, and analyze the data in order to offer possible solutions. Too often, we do not have patientcentred outcomes relevant to our question.

Desgranges et $_{\text {al. }}{ }^{2}$ state, "While there is some evidence that intraoperative hypothermia may contribute to perioperative morbidity in the general surgical population, there is a lack of data in the literature as to the potential consequences of maternal hypothermia during [Cesarean delivery] on perioperative maternal and neonatal outcomes." Interpretation of clinical trial outcomes needs 
to go beyond a simple discussion of statistical significance to include the relevance of the clinical problem in question.

In general, perioperative hypothermia may lead to increased risk for morbid cardiac events, postoperative wound infection, coagulopathy, intraoperative blood loss, transfusion requirement, and prolonged recovery, but we do not know if the same risks are present for our obstetric patients. We cannot necessarily extrapolate data from the colorectal, thoracic, orthopedic, or vascular surgical patients which have been referenced. ${ }^{4-6}$ These patients are not equivalent to those undergoing Cesarean deliveryyoung, generally healthy, in a physiological hypercoagulable state, undergoing a relatively short procedure almost exclusively with neuraxial anesthesia, and some labouring before entering the operating room.

In 2015, Sultan et al. published a meta-analysis synthesizing the evidence of the effect of patient warming on neonatal and maternal outcomes. ${ }^{7}$ These authors reviewed 13 studies and 789 patients and, with respect to maternal outcomes, found that active warming reduces hypothermia, reduces shivering, and improves thermal comfort. The authors of that study and the one by Desgranges et al. ${ }^{2}$ did not address the issue of relevance or the answer to the question of were there any improvements in patient-centred outcomes?

The goal of research is to improve patient care and/or quality of life. Despite this, research does not always consider the mutual interests of patients and healthcare institutions. We are trained in statistics and empirical evidence, while our patients and their families experience healthcare and tell their stories. Patients' priorities exist within these experiences and stories - anesthesia research needs to align with these priorities. Clinical trials should include clinically relevant patient-centred outcome measures rather than surrogate outcomes, which may have relevance only for the clinician/researcher. ${ }^{8}$

Researchers can look for guidance from various resources. The European Society of Anaesthesiology and European Society of Intensive Care Medicine jointly published "Standards for definitions and use of outcome measures for clinical effectiveness research in perioperative medicine". ${ }^{9}$ This statement defines and grades a set of robust patientrelated outcomes using a methodology of literature review followed by expert review and subsequent consensus. The COMPAC-StEP (Core Outcomes Measures in Perioperative and Anaesthetic Care - Standardized Endpoints for Perioperative Medicine) collaboration is defining a core set of perioperative outcomes through systematic review and formalized expert consensus via a Delphi process. ${ }^{10}$ Lastly, the Patient-Centered Outcomes Research Institute (PCORI) was established to promote comparative clinical effectiveness research that will "assist patients, clinicians, purchasers, and policy-makers in making informed health decisions". ${ }^{11}$

Perioperative anesthesia research must embrace bringing patients and clinicians together to prioritize questions in evaluating new therapies or treatment uncertainty. $^{12}$ The National Institute of Academic Anaesthesia had previously identified that the description of quality care in anesthesia was limited by a lack of valid measures. ${ }^{13}$ In collaboration with the James Lind Alliance, they brought together clinicians, patients, and caregivers to identify areas of practice where uncertainties exist and to prioritize clinical research questions to address these issues. The aim of such "priority setting partnerships" is to develop research questions using measurable outcomes that are of direct relevance to patients. ${ }^{14}$ Priority setting partnerships encourage organizational and institutional collaboration and avoid misuse of resources - e.g., by limiting unnecessary patient participation and duplication of research.

The patients we treat are partners in their healthcare; they decide to undergo surgery based on personal values. Patients want to know that their care will cure disease, improve health, or, as in the Desgranges et al. study, lead to the safe delivery of a newborn. They expect tolerable postoperative discomfort, minimal risk of complications, and rapid return of function the onus is on us to discover their priorities. We must now design trials with outcomes that genuinely reflect real-world settings and concerns and enable shared decision-making. Patient-centred outcome measures after anesthesia and surgery should include aspects of patient comfort (e.g., pain relief), quality of recovery (e.g., emotional and functional status), serious complications (e.g., relevant adverse events, ideally defined and measured in a standardized way), and disability-free survival (i.e., longer term recovery free of disability). ${ }^{8}$

Hypothermia may predispose our patients to infectious, hemorrhagic, or cardiac-related complications, but frankly, we just don't know how hypothermia impacts women having a Cesarean delivery. If forced-air warming techniques are to be used in the perioperative period for women having a Cesarean delivery, we should look for reliable and validated evidence to clarify the added value for these women. Measuring outcomes in healthcare can be complicated, and meaningful outcome measures should be validated and result in improved care or ensure that patients have a positive healthcare experience. In the current perioperative anesthesia research context (i.e., scrutinizing the value of medical research, enabling shared decisionmaking), we should re-focus. We must ask relevant questions and answer with meaningful outcomes - it's not that complicated. 
Nous devons poser des questions pertinentes et $y$ répondre avec des résultats significatifs

"Personne ne savait que les soins de santé pouvaient être si compliqués », voilà comment le président des États-Unis Donald Trump a décrit son expérience d'un mois de politiques de soins de santé lorsqu'il s'est adressé à un groupe de gouverneurs à la Maison Blanche le 27 février 2017. ${ }^{1}$

N'importe quelle personne travaillant dans les soins de santé aurait pu partager notre secret mal gardé avec le président Trump : les soins de santé sont compliqués! En fait, les soins de santé sont très compliqués et, dans certaines situations spécifiques, l'absence de critères d'évaluation et de mesures normalisés de résultats centrés sur le patient peut contribuer à cette complexité. Pour être pertinente, une mesure d'évaluation centrée sur le patient doit être validée, avoir une signification clinique (c.-à-d. une valeur personnelle pour le patient) et améliorer les soins ou favoriser une expérience positive des soins de santé - mais dans certaines situations, ces mesures peuvent devenir compliquées.

Dans ce numéro du Journal, Desgranges et coll. ${ }^{2}$ présentent les résultats d'une étude de cohorte prospective dans laquelle ils examinent les facteurs prédictifs d'hypothermie maligne pendant un accouchement par césarienne. Dans leur étude, les auteurs ont surveillé la température tympanique de femmes devant subir un accouchement par césarienne urgent ou non urgent. La température a été mesurée à l'arrivée en salle d'opération, à l'incision de la peau, puis à la fin de la fermeture de la peau. Les accouchements par césarienne réalisés sous anesthésie neuraxiale ou générale ont été inclus.

Après l'induction de l'anesthésie, on a gardé les patientes au chaud à l'aide d'une couverture en coton placée sur le haut du corps et à l'aide d'un système de réchauffement à air forcé réglé à $43^{\circ} \mathrm{C}$. L'hypothermie était définie en tant qu'une température tympanique $<36^{\circ} \mathrm{C}$ à la fin de la fermeture de la peau. Divers facteurs démographiques, chirurgicaux et anesthésiques préopératoires et peropératoires ont été enregistrés et analysés en tant que prédicteurs possibles d'hypothermie maternelle. Trois cents cinquante-neuf femmes ont été incluses dans l'analyse, et $23 \%$ de ces patients ont souffert d'hypothermie. L'analyse des chercheurs a montré que l'utilisation d'un système de réchauffement à air forcé actif était associée à une réduction du risque d'hypothermie maternelle, tandis qu'une température maternelle plus basse à l'arrivée en salle d'opération ainsi qu'un volume accru de liquides intraveineux non chauffés constituaient des prédicteurs d'hypothermie maternelle. Selon
Desgranges et coll., ces résultats devraient motiver l'anesthésiologiste à surveiller la température maternelle avant et pendant un accouchement par césarienne, étant donné que l'hypothermie maternelle peut entraîner de graves complications.

Toutefois, le lecteur devrait réfléchir à la question fondamentale suivante après avoir lu l'étude de Desgranges et coll. ${ }^{2}$ : Quelle est la pertinence du problème clinique de l'hypothermie maligne pendant un accouchement par césarienne pour la patiente? En appliquant les critères dits FINER (pour faisable, intéressant, nouveau, éthique et pertinent (relevant en anglais)), ${ }^{3}$ les chercheurs cliniques tentent de procurer des réponses. Lorsque confrontés à des problèmes cliniques, nous sommes formés à poser des questions, expliquer les faits et analyser les données afin de proposer des solutions possibles. Trop souvent, nous n'avons pas de résultats centrés sur le patient pertinents à notre question.

Desgranges et coll. ${ }^{2}$ déclarent : «Bien qu'il existe des données probantes selon lesquelles l'hypothermie peropératoire pourrait contribuer à la morbidité périopératoire dans la population chirurgicale générale, les données font défaut dans la littérature quant aux conséquences potentielles de l'hypothermie maternelle pendant un [accouchement par césarienne] sur les pronostics maternels et néonataux périopératoires. » Cependant, l'interprétation des résultats d'études cliniques doit aller plus loin qu'une simple discussion de la signification statistique et inclure la pertinence du problème clinique en question.

En général, l'hypothermie périopératoire peut entraîner un risque accru de complications cardiaques morbides, d'infection postopératoire de la plaie, de coagulopathie, de pertes de sang peropératoires, de besoins transfusionnels et de récupération prolongée, mais nous ne savons pas si ces mêmes risques s'appliquent à nos patientes obstétricales. Nous ne pouvons pas nécessairement extrapoler les données des patients de chirurgie colorectale, thoracique, orthopédique ou vasculaire qui sont cités en référence. ${ }^{4-6}$ Ces patients ne ressemblent pas aux patientes subissant un accouchement par césarienne - lesquelles sont jeunes, généralement en bonne santé, dans un état physiologique d'hypercoagulabilité, subissant une intervention relativement brève presque exclusivement sous anesthésie neuraxiale, et parfois en travail obstétrical avant d'entrer en salle d'opération.

En 2015, Sultan et coll. ont publié une méta-analyse résumant les données probantes concernant l'effet du réchauffement des patientes sur les pronostics néonataux et maternels. ${ }^{7}$ Ces auteurs ont passé en revue 13 études et 789 patientes et, en ce qui a trait aux pronostics maternels, ils ont découvert qu'un réchauffement actif réduisait 
l'hypothermie et les frissons et améliorait le confort thermique. Les auteurs de cette étude et de celle de Desgranges et coll. ${ }^{2}$ ne se sont pas intéressés à la question de la pertinence ou à la réponse à la question de savoir s'il $y$ avait une amélioration des résultats centrés sur le patient.

L'objectif de la recherche est d'améliorer les soins aux patients et/ou leur qualité de vie. Malgré cela, la recherche ne tient pas toujours compte des intérêts mutuels des patients et des institutions de soins de santé. Alors que nous sommes formés à réfléchir en statistiques et en données probantes empiriques, nos patients et leurs familles font l'expérience des soins de santé et racontent leurs histoires. Les priorités des patients existent donc dans le cadre de ces expériences et de ces histoires - et la recherche en anesthésie doit s'aligner sur ces priorités. Les études cliniques devraient inclure des mesures d'évaluation centrées sur le patient et pertinentes d'un point de vue clinique plutôt que des critères de substitution, qui pourraient n'être pertinents que pour le clinicien/chercheur. ${ }^{8}$

Les chercheurs peuvent s'inspirer de diverses ressources. La Société européenne d'anesthésiologie (ESA) et la Société européenne de réanimation (ESICM) ont publié conjointement un document intitulé « Normes pour définir et utiliser des mesures d'évaluation pour la recherche sur l'efficacité clinique en médecine périopératoire $»{ }^{9}$ Cet énoncé définit et classe un ensemble rigoureux de critères liés au patient à l'aide d'une méthodologie de revue de la littérature suivie d'une revue par un expert et d'un consensus subséquent. La collaboration COMPAC-StEP (Core Outcomes Measures in Perioperative and Anaesthetic Care - Standardized Endpoints for Perioperative Medicine, soit 'Mesures d'évaluation clés dans les soins périopératoires et anesthésiques - critères d'évaluation standardisés pour la médecine périopératoire') est en train de définir un ensemble central de critères périopératoires en réalisant une revue systématique et un consensus d'experts formalisés par un processus Delphi. ${ }^{10}$ Enfin, l'Institut PCORI (Patient-Centered Outcomes Research Institute Institut de recherche sur les critères centrés sur le patient) a été mis sur pied afin de promouvoir des recherches comparatives sur l'efficacité clinique qui «aideront les patients, les cliniciens, les acheteurs et les stratèges à prendre des décisions éclairées en matière de santé ». ${ }^{11}$

La recherche en anesthésie périopératoire doit accepter de réunir patients et cliniciens afin de prioriser les questions lors de l'évaluation de nouveaux traitements ou d'incertitude quant à un traitement. ${ }^{12}$ Par le passé, l'Institut national d'anesthésie universitaire (National Institute of Academic Anaesthesia) britannique avait déjà déclaré que la description de soins de qualité en anesthésie était confinée par l'absence de mesures valides. ${ }^{13}$ En collaboration avec l'Alliance James
Lind, l'Institut a réuni cliniciens, patients et personnel soignant afin d'identifier les domaines de la pratique où il existait des incertitudes et prioriser les questions de recherche clinique qui tentaient de répondre à ces questions. Le but de tels « partenariats de priorisation des objectifs » est de mettre au point des questions de recherche qui utilisent des critères mesurables directement pertinents pour les patients. ${ }^{14}$ Les partenariats de priorisation des objectifs encouragent la collaboration organisationnelle et institutionnelle et évitent le gaspillage des ressources, par exemple en limitant la participation inutile des patients et la duplication de la recherche.

Les patients que nous traitons sont des partenaires dans leurs soins de santé; ils décident de subir une chirurgie selon leurs propres valeurs. Les patients veulent savoir que les soins qu'ils reçoivent guériront leur maladie, amélioreront leur santé ou, comme dans l'étude de Desgranges et coll., entraîneront l'accouchement sécuritaire de leur nouveau-né. Ils s'attendent à un inconfort postopératoire tolérable, un risque minimal de complications, et un retour rapide de leurs fonctions - à nous de découvrir leurs priorités. Nous devons dorénavant concevoir des études visant des résultats qui reflètent véritablement les contextes et les préoccupations réels et permettre une prise de décision partagée. Les mesures d'évaluation centrées sur le patient après l'anesthésie et la chirurgie devraient inclure des aspects liés au confort du patient (par ex. le soulagement de la douleur), la qualité de la récupération (par ex. l'état fonctionnel et émotionnel), les complications graves (par ex. les effets secondaires néfastes pertinents, idéalement définis et mesurés de façon normalisée), et la survie sans handicap (c.-à-d. la récupération à long terme sans handicap). ${ }^{8}$

L'hypothermie pourrait prédisposer nos patientes à des complications infectieuses, hémorragiques ou cardiaques mais, pour être honnête, nous ne savons pas si l'hypothermie a un impact sur les femmes subissant un accouchement par césarienne. Si des techniques de réchauffement par air forcé sont utilisées en période périopératoire chez les femmes subissant un accouchement par césarienne, nous devrions chercher des données probantes fiables et validées afin de clarifier la valeur ajoutée de ces gestes pour ces femmes. La mesure des résultats peut être difficile en soins de santé, et les mesures de résultats significatives devraient être validées et entraîner une amélioration des soins ou garantir que les patients aient une expérience positive des soins de santé. Dans le contexte actuel de la recherche en anesthésie périopératoire (c.-à-d. l'examen minutieux de la valeur de la recherche médicale, la création de prise de décision partagée), nous devrions réorienter notre objectif. Nous devons poser des questions pertinentes et répondre avec des résultats significatifs - ce n'est pas si compliqué. 
Conflicts of interest None declared.

Editorial responsibility This submission was handled by Dr. Philip M. Jones, Associate Editor, Canadian Journal of Anesthesia.

\section{Conflit d'intérêt Aucun.}

Responsabilité éditoriale Cet article a été traité par Dr Philip M. Jones, rédacteur adjoint, Journal canadien d'anesthésie.

\section{References}

1. Lizza $R$. Donald Trump learns that reforming healthcare is "complicated". The New Yorker - February 28 2017. Available from URL: http://www.newyorker.com/news/ryan-lizza/donaldtrump-learns-that-reforming-health-care-is-complicated (accessed May 2017).

2. Desgranges FP, Bapteste L, Riffard C, et al. Predictive factors of maternal hypothermia during cesarean delivery: a prospective cohort study. Can J Anesth 2017; 64: DOI:10.1007/s12630-017-0912-2.

3. Hulley SB, Cummings SR, Browner WS, Grady DG, Newman TB. Designing Clinical Research. 3rd ed. Philadelphia, PA: Lippincott Williams \& Wilkins; 2007 .

4. Frank SM, Fleisher LA, Breslow MJ, et al. Perioperative maintenance of normothermia reduces the incidence of morbid cardiac events. A randomized clinical trial. JAMA 1997; 277: 1127-34.

5. Kurz A, Sessler DI, Lenhardt R. Perioperative normothermia to reduce the incidence of surgical-wound infection and shorten hospitalization. Study of Wound Infection and Temperature Group. N Engl J Med 1996; 334: 1209-15.
6. Schmied H, Kurz A, Sessler DI, Kozek S, Reiter A. Mild hypothermia increases blood loss and transfusion requirements during total hip arthroplasty. Lancet 1996; 347: 289-92.

7. Sultan P, Habib AS, Cho Y, Carvalho B. The effect of patient warming during caesarean delivery on maternal and neonatal outcomes: a meta-analysis. Br J Anaesth 2015; 115: 500-10.

8. Myles PS. Perioperative outcomes: are we asking the right questions? Can J Anesth 2016; 63: 138-41.

9. Jammer I, Wickboldt N, Sander M, et al. Standards for definitions and use of outcome measures for clinical effectiveness research in perioperative medicine: European Perioperative Clinical Outcome (EPCO) definitions: a statement from the ESAESICM joint taskforce on perioperative outcome measures. Eur J Anaesthesiol 2015; 32: 88-105.

10. Myles PS, Grocott MP, Boney O. Moonesinghe SR; COMPACStEP Group. Standardizing end points in perioperative trials: towards a core and extended outcome set. Br J Anaesth 2016; 116: 586-9.

11. Selby JV, Beal AC, Frank L. The Patient-Centered Outcomes Research Institute (PCORI) national priorities for research and initial research agenda. JAMA 2012; 307: 1583-4.

12. Tallon D, Chard J, Dieppe P. Relation between agendas of the research community and the research consumer. Lancet 2000; 355: 2037-40.

13. The National Institute of Academic Anaesthesia. National Institute of Academic Anaesthesia Comprehensive Review 2008-2011 London, UK - 2012. Available from URL: https:// www.vasgbi.com/downloads/NIAA_Feb2012_Final.pdf (accessed May 2017).

14. Heneghan $C$, Goldacre B, Mahtani KR. Why clinical trial outcomes fail to translate into benefits for patients. Trials 2017; 18: 122 . 